\title{
Grouping of Xanthomonas campestris pathovars by SDS-PAGE of proteins
}

\author{
L. VAUTERIN, ${ }^{*}$ J. SwingS and K. Kersters \\ Laboratorium voor Microbiologie en microbiële Genetica, Ledeganckstraat 35, Rijksuniversiteit, 9000 Gent, Belgium
}

(Received 18 December 1990; revised 5 March 1991; accepted 25 March 1991)

\begin{abstract}
A numerical analysis was performed on SDS-PAGE protein patterns of 307 Xanthomonas strains comprising all species and $27 X$. campestris pathovars. The electrophoretic groupings corresponded in some, but not all cases with the existing pathovars. Six pathovars constituted distinct entities, comprising all strains investigated. These included $X$. campestris pv. campestris, $X$. campestris pv. graminis, $X$. campestris pv. hyacinthi, $X$. campestris pv. pelargonii, $X$. campestris pv. pruni and $X$. campestris pv. theicola. A great number of pathovars consisted of a homogeneous group from which only one or a few strains were aberrant. In two cases $(X$. campestris pv. ricini and $X$. campestris pv. vitians) even the pathovar reference strain was aberrant. Six pathovars from members of the plant family Fabaceae could not be differentiated from one another: $X$. campestris pv. phaseoli, X. campestris pv. phaseoli var. fuscans, $X$. campestris pv. cajani, $X$. campestris pv. vignicola, $X$. campestris pv. alfalfae and $X$. campestris pv. glycines. At least six $X$. campestris pathovars were heterogeneous, displaying two or more protein electrophoretic types: $X$. campestris pv. alfalfae, $X$. campestris pv. dieffenbachiae, $X$. campestris pv. juglandis, $X$. campestris pv. poinsettiicola, $X$. campestris pv. vesicatoria and $X$. campestris pv. vignicola. A database of SDS-protein patterns provides a valuable tool for the identification of unknown xanthomonads.
\end{abstract}

\section{Introduction}

The bacterial genus Xanthomonas consists of numerous plant pathogens, occurring worldwide and causing disease on diverse economically important crops (Bradbury, 1984; Kennedy \& Alcorn, 1980; Leyns et al., 1984). Whereas the determination of the genus Xanthomonas and its species poses no great problem, the characterization of the $X$. campestris pathovars is still difficult (Ercolani, 1987; Vauterin et al., 1990 b). In phytopathological practice, however, there is a need for rapid and unambiguous identification of pathovars of $X$. campestris. These pathovars, which are defined by host or symptom specificity, can usually not be differentiated by other phenotypic features (Dye, 1962; Van den Mooter \& Swings, 1990). A number of other techniques have been applied to differentiate pathovars of $X$. campestris, such as typing with polyclonal and monoclonal antibodies, gas chromatographic analysis of cellular fatty acids, protein electrophoresis and DNA probe techniques. Development of rapid high-resolution fingerprinting techniques such as protein electrophoresis in combination with computer-assisted processing of profiles makes it possible to characterize and compare large numbers of strains at the infrasubspecific level (Kersters, 1985;
Jackman, 1987). Protein electrophoresis was first used to differentiate Xanthomonas strains by El-Sharkawy \& Huisingh (1971). Later, protein electrophoretic studies of Xanthomonas allowed differentiation of $X$. campestris pv. oryzae and pv. oryzicola (Vera Cruz et al., 1984), pv. manihotis and pv. cassavae (Van den Mooter et al., 1987a), and pv. graminis, pv. phleipratensis, pv. poae and pv. arrhenatheri (Van den Mooter et al., 1987b). Van Zyl \& Steyn (1990) used SDS-PAGE to differentiate Pseudomonas and Xanthomonas species. A good agreement between groupings obtained by SDS-PAGE, gas chromatographic analysis of cellular fatty acids and DNA-DNA hybridization was found for a number of $X$. campestris pathovars (Kersters et al., 1989; Stead, 1989; Vauterin et al., 1990b).

In our laboratory, a database of patterns of proteins separated by SDS-PAGE of Xanthomonas has been generated, now comprising over 900 strains. In the present paper we report on a numerical comparison of electrophoretic protein patterns of 307 strains comprising $27 X$. campestris pathovars and representative strains of the other Xanthomonas species. Attention was paid to the inclusion of isolates representing the broad geographical distribution and phytopathological diversity of each pathovar. The main objectives of this work were (1) 
to examine whether the groupings obtained by SDSPAGE support the existing pathovar division of $X$. campestris as established by Dye et al. (1980); (2) to check the homogeneity or heterogeneity of each of the pathovars studied, and (3) to constitute a database of SDS-PAGE protein patterns on computer files for rapid identification.

\section{Methods}

Bacterial cells. All the strains used are listed in Table 1. For each of the species and pathovars studied, the type strain or pathovar reference strain was included. Strains were grown on GYCA medium (Dye, $1962)$, modified as follows: $1 \%(\mathrm{w} / \mathrm{v})$ glucose, $0.5 \%(\mathrm{w} / \mathrm{v})$ yeast extract, $3 \%(\mathrm{w} / \mathrm{v}) \mathrm{CaCO}_{3}$, and $2 \%(\mathrm{w} / \mathrm{v})$ agar in distilled water. Stock cultures were maintained at $2{ }^{\circ} \mathrm{C}$.

SDS-PAGE of whole-cell proteins. Cultures were grown on GYCA slants for $48 \mathrm{~h}$ at $28^{\circ} \mathrm{C}$. Cells were suspended in $0.01 \mathrm{M}$-potassium phosphate buffer $\mathrm{pH} 7.0$ and transferred to Roux flasks containing $150 \mathrm{ml} \mathrm{GYCA}$ medium. Flasks were incubated for $48 \mathrm{~h}$ at $28^{\circ} \mathrm{C}$.

Preparation and electrophoresis of whole-cell protein extracts was performed by the SDS-PAGE method of Laemmli (1970), using a slightly modified procedure. Cells were harvested from the flasks with $20 \mathrm{ml}$ phosphate-buffered saline (4.26 $\mathrm{g} \mathrm{Na}_{2} \mathrm{HPO}_{4} \mathrm{l}^{-1}, 2 \cdot 27 \mathrm{~g} \mathrm{KH}_{2} \mathrm{PO}_{4}$ $1^{-1}, 8 \mathrm{~g} \mathrm{NaCl}^{-1}$, in distilled water), and washed and centrifuged at 15000 r.p.m. twice. About $70-80 \mathrm{mg}$ of the pellet was suspended in sample treatment buffer $\left(7.5 \mathrm{~g}\right.$ Tris $1^{-1}, 50 \mathrm{ml} \beta$-mercaptoethanol $\mathrm{1}^{-1}$, $100 \mathrm{ml}$ glycerol $\mathrm{l}^{-1}$, in double-distilled water; final $\mathrm{pH} 6.8$, adjusted with $\mathrm{HCl}$ ) in an Eppendorf tube; $0 \cdot 14 \mathrm{ml}$ of a $20 \%$ (w/v) SDS solution was added, and the suspension was mixed and heated at $95^{\circ} \mathrm{C}$ for $10 \mathrm{~min}$. Tubes were cooled on ice and centrifuged at 10000 r.p.m. Supernatant extracts were divided into two portions, one of which was stored at $-20^{\circ} \mathrm{C}$ for immediate use and the other at $-80^{\circ} \mathrm{C}$ for long storage.

Electrophoresis was performed in a $12 \%(\mathrm{w} / \mathrm{v})$ gel slab, run vertically $\left(6 \mathrm{~mA}\right.$ constant current, $19^{\circ} \mathrm{C}$ ) until the bromophenol blue tracking dye had migrated $95 \mathrm{~mm}$ (about $15 \mathrm{~h}$ ). Gels were stained for $1 \mathrm{~h}$ in a solution containing $0.25 \%$ (w/v) Serva Blue R, $50 \%$ (v/v) ethanol and $10 \%(\mathrm{v} / \mathrm{v})$ acetic acid, and destained in a solution containing $25 \%$ methanol and $10 \%$ acetic acid. Gels were dried in a Bio-Rad 224 slab gel dryer.

The reproducibility of the electrophoresis technique was monitored by preparing protein extracts of over 40 strains in duplicate and running reference samples on each of the slab gels.

Densitometric recording and normalization of electrophoregrams. The dried stained gels were scanned with an LKB laser densitometer linked to a microcomputer. Densitometric records of 1000 points were normalized and reduced to 400 points as described by Pot et al. (1989).

Secondary alignment of traces and numerical analysis. Numerical analysis was performed on a Siemens BS2000 mainframe computer. A Fortran 77 program was developed to perform numerical analysis of up to 800 traces (L. Vauterin, unpublished data). The program calculated the similarity between traces by the Pearson product-moment correlation coefficient $(r)$ (Sneath \& Sokal, 1973). To optimize matching between pairs of traces, the program performed a secondary alignment of traces during numerical analysis. This alignment involved a lateral displacement of one trace up to five points on either side of the initial alignment. The highest $r$-value was retained. For a given pair of traces, the procedure involved the following steps: (1) calculation of the initial correlation at the original alignment; (2) displacement of one trace with respect to the other by a single point step forward and recalculation of the correlation; (3) if the new correlation was higher than the initial one, further single-point steps forward were performed until the maximum of correlation was reached. If the correlation value obtained in (2) was lower than the initial one, the routine was executed again from step (2) backward from the original alignment.

Cluster analysis was performed on the matrix of correlation values by the unweighted pair group method using arithmetic averages (UPGMA).

\section{Results and Discussion}

The secondary alignment of the standardized traces during numerical analysis improved the reliability of the cluster analysis considerably, even though the protein patterns were highly standardized. An increase of correlation of $17 \%$ was observed between patterns of $X$. campestris pv. hyacinthi strains. A similar optimization has been applied by other investigators (Albritton et al., 1988; Costas et al., 1989; Jackman et al., 1983). The overall reproducibility of the electrophoresis technique averaged $r=0.94$.

The dendrogram obtained after numerical analysis of protein electrophoregrams of 307 Xanthomonas strains is shown in Fig. 1. In this simplified version, homogeneous groups are represented by triangles. Nineteen clusters of at least three strains clustering at $r=0.85$ were delineated. Strains and corresponding clusters are listed in Table 1. In the discussion hereafter the location of the pathovar reference strain is only mentioned when it grouped apart from its electrophoretic cluster, or when the pathovar consisted of different clusters.

From the fairly high similarity values between clusters of Xanthomonas species and $X$. campestris pathovars it appears that most of the xanthomonads display a common SDS-PAGE protein profile (see also the electrophoregrams in Fig. 2). Highly distinct profiles were only produced by $X$. albilineans and $X$. maltophilia. The species $X$. axonopodis, $X$. fragariae and $X$. oryzae (both pv. oryzae and pv. oryzicola) could be easily distinguished from each other and from the $X$. campestris pathovars, whereas $X$. populi was rather similar to $X$. campestris.

From $286 X$. campestris strains studied, 253 (i.e. $88 \%$ ) grouped together above $r=0.70$ (clusters 1 to 12 ). Clearly distinct from this group were $X$. campestris pv. melonis (cluster 19), pv. graminis Egli \& Schmidt 1982 (cluster 17) and pv. theicola (cluster 16).

$X$. campestris pv. graminis Egli \& Schmidt 1982 is a remarkably distinct and homogeneous pathovar. In previous studies this pathovar could be distinguished from other $X$. campestris pathovars including xanthomonads from grasses and cereals (Van den Mooter \& Swings, 1990; Van den Mooter et al., 1987a). All strains 
Table 1. Source and cluster assignment of the strains studied

ATCC, American Type Culture Collection, Rockville, Maryland, USA; CNBP, Collection Nationale de Bactéries Phytopathogènes, Angers, France; ICMP, International Collection of Microorganisms from Plants, Auckland, New Zealand; ICPB, International Collection of Phytopathogenic Bacteria, Davis, California; IPO, Instituut voor Plantenziektenkundig onderzoek, Wageningen, The Netherlands; LMG, Laboratorium voor Microbiologie Gent Culture Collection, Gent, Belgium; NCPPB, National Collection of Plant Pathogenic Bacteria, Harpenden, UK; PD, Plantenziektenkundige Dienst, Wageningen, The Netherlands.

\begin{tabular}{|c|c|}
\hline Species/pathovar & Strains \\
\hline \multicolumn{2}{|l|}{$X$. albilineans } \\
\hline Unclustered & $\begin{array}{l}\text { LMG } 487 \text { (NCPPB 1050), LMG } 490 \text { (NCPPB 2247), LMG } 494^{\mathrm{T}} \text { (NCPPB } \\
\text { 2969) }\end{array}$ \\
\hline \multicolumn{2}{|r|}{ - } \\
\hline Unclustered & LMG 538 (NCPPB 457), LMG 540 (NCPPB 2376), LMG 537 (ICMP 74) \\
\hline \multicolumn{2}{|c|}{$X$. campestris pv. alfalfae } \\
\hline Cluster 3a & LMG 8019 (NCPPB 1821), LMG 8020 (NCPPB 2393) \\
\hline Cluster $3 \mathrm{c}$ & $\begin{array}{l}\text { LMG } 8078 \text { (ICMP 330), LMG } 8079 \text { (ICMP 3376), LMG } 8080 \text { (ICMP } \\
4765 \text { ) }\end{array}$ \\
\hline Unclustered & $\begin{array}{l}\text { LMG } 495 \text { (ICPB XA121), LMG } 497^{*} \text { (NCPPB 2062), LMG } 8018 \text { (NCPPB } \\
480 \text { ) }\end{array}$ \\
\hline \multicolumn{2}{|c|}{$X$. campestris pv. begoniae } \\
\hline Cluster 11 & $\begin{array}{l}\text { LMG } 552 \text { (NCPPB 2605), LMG } 7190 \text { (NCPPB 1925), LMG } 7194 \text { (CNBP } \\
\text { 1422), LMG } 7196 \text { (CNBP 1424), LMG } 7594 \text { (Vantomme Xb1809), LMG } \\
7596 \text { (Vantomme Xb3007), LMG } 7597 \text { (Vantomme Xb2208), LMG } 7595 \\
\text { (Vantomme Xb1207), LMG } 7226 \text { (ATCC 8718), LMG } 7193 \text { (CNBP 1421), } \\
\text { LMG } 7227 \text { (ATCC 11726) }\end{array}$ \\
\hline Unclustered & $\begin{array}{l}\text { LMG 7303* (ICMP 194), LMG } 7307 \text { (ICMP 6652), LMG } 7188 \text { (NCPPB } \\
\text { 109), LMG } 7304 \text { (ICMP 195) }\end{array}$ \\
\hline \multicolumn{2}{|c|}{$X$. campestris pv. cajani } \\
\hline Cluster 3a & $\begin{array}{l}\text { LMG 558* (NCPPB 573), LMG } 7387 \text { (NCPPB 2063), LMG } 7473 \text { (ICMP } \\
\text { 1625) }\end{array}$ \\
\hline \multicolumn{2}{|c|}{$X$. campestris pv. campestris } \\
\hline Cluster 1 & $\begin{array}{l}\text { LMG } 8121 \text { (ICMP 6537), LMG } 8100 \text { (ICMP 4013), LMG } 8123 \text { (ICMP } \\
\text { 6542), LMG 8099 (ICMP 3984), LMG 583 (Maraite HMB142), LMG } \\
\text { 8051 (PD 923), LMG 8003 (NCPPB 1043), LMG 8035 (CFBP 1869), } \\
\text { LMG 8102 (ICMP 4578), LMG 8001 (NCPPB 529), LMG 7516 (ICMP } \\
\text { 5566), LMG 8112 (ICMP 5571), LMG 571 (NCPPB 1144), LMG 568 } \\
\text { (NCPPB 528), LMG } 7662 \text { (Vantomme Xc0611) }\end{array}$ \\
\hline \multicolumn{2}{|c|}{$X$. campestris pv. cassavae } \\
\hline Cluster 5 & $\begin{array}{l}\text { LMG } 8048 \text { (PD 771), LMG 8049 (PD 776), LMG } 670 \text { (ICPB XC110), } \\
\text { LMG 673* (Maraite HMB54), LMG 671 (Maraite HMB29), LMG 672 } \\
\text { (Maraite HMB38), LMG 764 (Maraite HMB59), LMG 5268 (Maraite } \\
\text { HMB45), LMG 5269 (Maraite HMB51), LMG 5270 (Maraite HMB53), } \\
\text { LMG 5246 (Maraite HMB179), LMG 5265 (Maraite HMB41), LMG 5264 } \\
\text { (Maraite HMB37), LMG 5267 (Maraite HMB44) }\end{array}$ \\
\hline Unclustered & LMG 5271 (Maraite HMB146) \\
\hline \multicolumn{2}{|c|}{$X$. campestris pv. citri } \\
\hline Cluster 6 & $\begin{array}{l}\text { LMG 682* (NCPPB 409), LMG } 680 \text { (NCPPB 405), LMG } 683 \text { (NCPPB } \\
\text { 410), LMG 681 (NCPPB 408), LMG } 8650 \text { (ICMP 28), LMG } 8652 \text { (ICMP } \\
\text { 594), LMG 8653 (ICMP 5809), LMG } 8654 \text { (ICMP 7494), LMG } 8657 \\
\text { (ICMP 8436), LMG 8651 (ICMP 31), LMG 9176 (Civerolo XC59), LMG } \\
9177 \text { (Civerolo XC62), LMG } 9184 \text { (Civerolo XC115) }\end{array}$ \\
\hline Unclustered & LMG 8655 (ICMP 8432), LMG 8656 (ICMP 8435) \\
\hline \multicolumn{2}{|c|}{$X$. campestris pv. cucurbitae } \\
\hline Cluster 3b & LMG 7479 (ICMP 203) \\
\hline Cluster 10 & $\begin{array}{l}\text { LMG } 7480 \text { (ICMP 1858), LMG } 7481 \text { (ICMP 4766), LMG 690* (NCPPB } \\
\text { 2597), LMG } 8662 \text { (ICMP 2309), LMG } 8661 \text { (ICMP 202), LMG } 8663 \\
\text { (ICMP 4769) }\end{array}$ \\
\hline \multicolumn{2}{|c|}{$X$. campestris $\mathrm{pv}$. dieffenbachiae } \\
\hline Cluster $3 \mathrm{e}$ & LMG 7399 (NCPPB 985), LMG 7400 (NCPPB 986) \\
\hline Cluster 8 & $\begin{array}{l}\text { LMG } 695^{*} \text { (NCPPB 1833), LMG } 7484 \text { (ICMP 4656), LMG } 8664 \text { (ICMP } \\
7461)\end{array}$ \\
\hline
\end{tabular}


Table 1-contd.

\begin{tabular}{|c|c|}
\hline Species/pathovar & Strains \\
\hline \multicolumn{2}{|c|}{$X$. campestris pv. glycines } \\
\hline Cluster 3a & $\begin{array}{l}\text { LMG } 712^{*} \text { (NCPPB 554), LMG } 7403 \text { (NCPPB 1124), LMG } 8023 \text { (NCPPB } \\
\text { 1135), LMG 7404 (NCPPB 1717), LMG } 8026 \text { (NCPPB 1714), LMG } 8025 \\
\text { (NCPPB 1141), LMG 8125 (ICMP 246), LMG } 8126 \text { (ICMP 3415), LMG } \\
8127 \text { (ICMP 3514), LMG } 7488 \text { (ICMP 3434) }\end{array}$ \\
\hline Cluster 7 & LMG 8027 (NCPPB 1716) \\
\hline Unclustered & LMG 8128 (ICMP 3536) \\
\hline \multicolumn{2}{|c|}{ X. campestris pv. graminis Egli \& Schmidt 1982} \\
\hline Cluster 17 & $\begin{array}{l}\text { LMG } 726^{*} \text { (NCPPB 2700), LMG } 607 \text { (Leyns Xg81-28), LMG } 713 \text { (Van den } \\
\text { Mooter 218), LMG } 600 \text { (Leyns Xg81-16), LMG 587 (IPO 118), LMG 595 } \\
\text { (Egli E741), LMG } 7406 \text { (NCPPB 2696), LMG } 7408 \text { (NCPPB 2701), LMG } \\
593 \text { (Egli E733), LMG } 7407 \text { (NCPPB 2698) }\end{array}$ \\
\hline \multicolumn{2}{|c|}{$X$. campestris pv. hederae } \\
\hline Cluster $7 \mathrm{e}$ & $\begin{array}{l}\text { LMG 733* (NCPPB 939), LMG } 734 \text { (ICPB XH1), LMG } 7414 \text { (NCPPB } \\
\text { 2336), LMG } 7413 \text { (NCPPB 2016), LMG } 8666 \text { (ICMP 1663), LMG } 8665 \\
\text { (ICMP 1406) }\end{array}$ \\
\hline Unclustered & $\begin{array}{l}\text { LMG } 7411 \text { (NCPPB 987), LMG } 8668 \text { (ICMP 9510), LMG } 8667 \text { (ICMP } \\
\text { 9508) }\end{array}$ \\
\hline \multicolumn{2}{|c|}{$X$. campestris pv hyacinthi } \\
\hline Cluster 4 & $\begin{array}{l}\text { LMG } 7419 \text { (NCPPB 205), LMG 739* (NCPPB 599), LMG } 740 \text { (NCPPB } \\
\text { 1622), LMG } 7420 \text { (NCPPB 2183), LMG } 8041 \text { (PD 205), LMG } 8044 \text { (PD } \\
\text { 281), LMG } 8042 \text { (PD 357), LMG } 8043 \text { (PD 280), LMG } 742 \text { (ICPB } \\
\text { XH110), LMG } 741 \text { (NCPPB 2095) }\end{array}$ \\
\hline \multicolumn{2}{|c|}{$X$. campestris pv. juglandis } \\
\hline Cluster 2 & LMG 8045 (PD 130), LMG 8046 (PD 157), LMG 8047 (PD 189) \\
\hline Cluster $7 \mathrm{a}$ & $\begin{array}{l}\text { LMG } 751 \text { (NCPPB 1447), LMG } 748 \text { (NCPPB 412), LMG } 750 \text { (NCPPB } \\
\text { 414), LMG } 752 \text { (NCPPB 1659), LMG } 746 \text { (NCPPB 362), LMG } 747^{*} \\
\text { (NCPPB 411) }\end{array}$ \\
\hline \multicolumn{2}{|c|}{$X$. campestris pv. malvacearum } \\
\hline Cluster $3 \mathrm{e}$ & LMG 7430 (NCPPB 1699), LMG 7428 (NCPPB 1697) \\
\hline Cluster $3 f$ & $\begin{array}{l}\text { LMG } 759 \text { (Maraite HMB24), LMG } 761^{*} \text { (NCPPB 633), LMG } 760 \\
\text { (NCPPB 210), LMG 7429 (NCPPB 1698), LMG } 7427 \text { (NCPPB 1048), } \\
\text { LMG } 762 \text { (NCPPB 2837), LMG } 764 \text { (NCPPB 2840), LMG } 763 \text { (NCPPB } \\
\text { 2838) }\end{array}$ \\
\hline Cluster $7 \mathrm{c}$ & LMG 7426 (NCPPB 293) \\
\hline \multicolumn{2}{|c|}{$X$. campestris pv. manihotis } \\
\hline Cluster 9 & $\begin{array}{l}\text { LMG } 784 \text { (Maraite HMB55), LMG } 766 \text { (Maraite HMB3), LMG } 769 \\
\text { (Maraite HMB10), LMG } 779 \text { (Maraite HMB80), LMG } 767 \text { (Maraite } \\
\text { HMB6), LMG } 782 \text { (Maraite HMB93), LMG } 768 \text { (Maraite HMB9), LMG } \\
783 \text { (Maraite HMB148), LMG } 780 \text { (Maraite HMB81), LMG } 771 \text { (Maraite } \\
\text { HMB25), LMG } 777 \text { (Maraite HMB78), LMG 773* (NCPPB 1834), LMG } \\
774 \text { (Maraite HMB60) }\end{array}$ \\
\hline Unclustered & LMG 778 (Maraite HMB79) \\
\hline \multicolumn{2}{|c|}{$X$. campestris pv. melonis } \\
\hline Cluster 19 & $\begin{array}{l}\text { LMG } 8673 \text { (ICMP 8688), LMG 8670* (ICMP 8682), LMG } 8672 \text { (ICMP } \\
8685 \text { ) }\end{array}$ \\
\hline Unclustered & LMG 8671 (ICMP 8683), LMG 8674 (ICMP 9284) \\
\hline \multicolumn{2}{|c|}{$X$. campestris pv. pelargonii } \\
\hline Cluster 12 & $\begin{array}{l}\text { LMG } 7708 \text { (Vantomme Xp1025), LMG } 7691 \text { (Vantomme Xp1000), LMG } \\
7317 \text { (ICMP 5919), LMG } 7715 \text { (Vantomme Xp1031), LMG } 7318 \text { (ICMP } \\
\text { 6758), LMG } 7690 \text { (Vantomme Xp0904), LMG } 7721 \text { (Vantomme Xp1039), } \\
\text { LMG } 7763 \text { (Vantomme Xp1040), LMG } 7764 \text { (Vantomme Xp1041), LMG } \\
7312 \text { (ICMP 230), LMG } 7353 \text { (ICMP 229), LMG } 7315 \text { (ICMP 5909), } \\
\text { LMG } 7316 \text { (ICMP 5915), LMG } 7354 \text { (ICMP 241), LMG 7314* (ICMP } \\
\text { 4321) }\end{array}$ \\
\hline \multicolumn{2}{|c|}{$X$. campestris pv. phaseoli } \\
\hline Cluster $3 \mathrm{a}$ & $\begin{array}{l}\text { LMG } 842 \text { (Saettler XpNcb), LMG } 8011 \text { (NCPPB 1646), LMG } 8015 \\
\text { (NCPPB 2064), LMG 7455* (NCPPB 3035), LMG 822 (Saettler Xp12), } \\
\text { LMG } 823 \text { (Saettler Xp21), LMG } 827 \text { (IPO 382), LMG } 836 \text { (NCPPB 1420), } \\
\text { LMG } 8014 \text { (NCPPB 1811) }\end{array}$ \\
\hline Cluster $3 b$ & $\begin{array}{l}\text { LMG } 830 \text { (NCPPB 557), LMG } 821 \text { (Saettler Xp11), LMG } 829 \text { (NCPPB } \\
556 \text { ) }\end{array}$ \\
\hline
\end{tabular}


Table 1-contd.

\begin{tabular}{lc}
\hline \hline Species/pathovar & Strains \\
\hline
\end{tabular}

X. campestris pv. phaseoli (var. fuscans)

Cluster $3 \mathrm{a}$

LMG 8036 (CFBP 1505), LMG 8037 (CFBP 1815), LMG 8017 (NCPPB 1495), LMG 7456 (NCPPB 456), LMG 7457 (NCPPB 1056), LMG 7458 (NCPPB 1158), LMG 8016 (NCPPB 1433), LMG 824 (PD 24), LMG 8038 (PD 24), LMG 8040 (PD 730), LMG 8039 (PD 365), LMG 841 (NCPPB 2665), LMG 832 (NCPPB 670), LMG 7512 (ICMP 5807), LMG 838 (NCPPB 2033), LMG 826 (NCPPB 381), LMG 7459 (NCPPB 1402), LMG 837 (NCPPB 1654)

Unclustered

LMG 7511 (ICMP 242), LMG 825 (Maraite HMB144), LMG 666 (Saettler Xpfwv)

$X$. campestris pv. pisi Unclustered

LMG $847^{*}$ (NCPPB 762)

$X$. campestris pv. poinsettiicola Cluster 3c Cluster 7c

Cluster 19

Unclustered

$X$. campestris pv. pruni Cluster 7c

LMG 8677 (ICMP 9512), LMG 8678 (ICMP 9513)

LMG 5402 (ICMP 3279)

LMG 7436 (NCPPB 1939), LMG 8675 (ICMP 3280)

LMG 5403 (ICMP 6274), LMG 8676 (ICMP 7180), LMG 849* (ICPB XP137), LMG 5401 (ICMP 332)

LMG 850 (ICPB XP10), LMG 852* (NCPPB 416), LMG 851 (NCPPB 273), LMG 853 (NCPPB 418), LMG 8681 (ICMP 6677), LMG 854 (NCPPB 419), LMG 856 (NCPPB 923), LMG 855 (NCPPB 420), LMG 857 (NCPPB 924), LMG 858 (NCPPB 926), LMG 8679 (ICMP 86), LMG

$X$. campestris pv. ricini Cluster 3c 7437 (NCPPB 272), LMG 7438 (NCPPB 368), LMG 8680 (ICMP 4100)

LMG 862 (NCPPB 1324), LMG 864 (NCPPB 2065), LMG 7442 (NCPPB 535), LMG 7443 (NCPPB 1473), LMG 7444 (NCPPB 1684), LMG 7440 (NCPPB 113), LMG 8683 (ICMP 7463)

Unclustered

$X$. campestris pv. theicola Cluster 16

LMG 861* (NCPPB 1063), LMG 7441 (NCPPB 488), LMG 8682 (ICMP 250)

LMG 8684* (ICMP 6774), LMG 8685 (ICMP 7291), LMG 8686 (ICMP 7294)

$X$. campestris pv. vesicatoria Cluster 3d

LMG 667 (ICMP 4799), LMG 668 (ICMP 6061), LMG 909 (Maraite HMB108), LMG 910 (Maraite HMB128), LMG 913 (Maraite HMB140), LMG 905 (Kishun 82-2), LMG 914 (Maraite HMB141), LMG 906 (Kishun 82-3), LMG 922 (NCPPB 941), LMG 929 (NCPPB 2573)

Cluster 7

Cluster $7 b$ LMG 920 (NCPPB 701)

LMG 917 (NCPPB 424), LMG 916 (NCPPB 423), LMG 935 (ICMP 118), LMG 925 (NCPPB 1431), LMG 911* (Maraite HMB 130), LMG 919 (NCPPB 509)

$X$. campestris pv. vignicola Cluster 3a

Cluster 3e

LMG 828 (NCPPB 555), LMG 831 (NCPPB 638), LMG 839 (NCPPB 2059), LMG 840 (NCPPB 2061), LMG 8138 (ICMP 590)

LMG 8136 (ICMP 334), LMG 8137 (ICMP 335), LMG 8139 (ICMP 3389), LMG 8140 (ICMP 3479), LMG 8752* (NCPPB 1838)

X. campestris pv. vitians Cluster 6 Cluster 7d

LMG 8689 (ICMP 6656), LMG 937* (NCPPB 976); LMG $\$ 38$ (NCPPB 2248), LMG 7452 (NCPPB 970), LMG 7508 (ICMP 4165, LMG 8687 (ICMP 355), LMG 7509 (ICMP 5854), LMG 8688 (ICMP 6461), LMG 8690 (ICMP 6857), LMG 7510 (ICMP 6655)

$X$. fragariae
Cluster 18

X. maltophilia
Unclustered
X. oryzae pv. oryzae
Cluster 14
X. oryzae pv. oryzicola
Cluster 15
X. populi
Cluster 13

LMG 707 (Benaki F8), LMG $708^{\mathrm{T}}$ (NCPPB 1469), LMG 709 (NCPPB 1822)

LMG 978 (NCPPB 323), LMG 958' (ATCC 13637), LMG 961 (ATCC 13636)

LMG 5047 (ICMP 3125), LMG 9602 (PXO 35), LMG 641 (ICMP 4646)

LMG 797* (NCPPB 1585), LMG 9646 (BLS 127), LMG 661 (ICMP 3134)

LMG 5743 (CNBP 1817), LMG 5751 (CNBP 1830), LMG 5750 (CNBP 1829)

* Pathovar reference strain.

T Type strain. 


\begin{tabular}{rrrrrrrr}
$r$ & & & \\
1.0 & 0.9 & 0.8 & 0.7 & 0.6 & 0.5 & 0.4 & 0.3 \\
\hline & 1 & 1 & 1 & 1 & 1
\end{tabular}

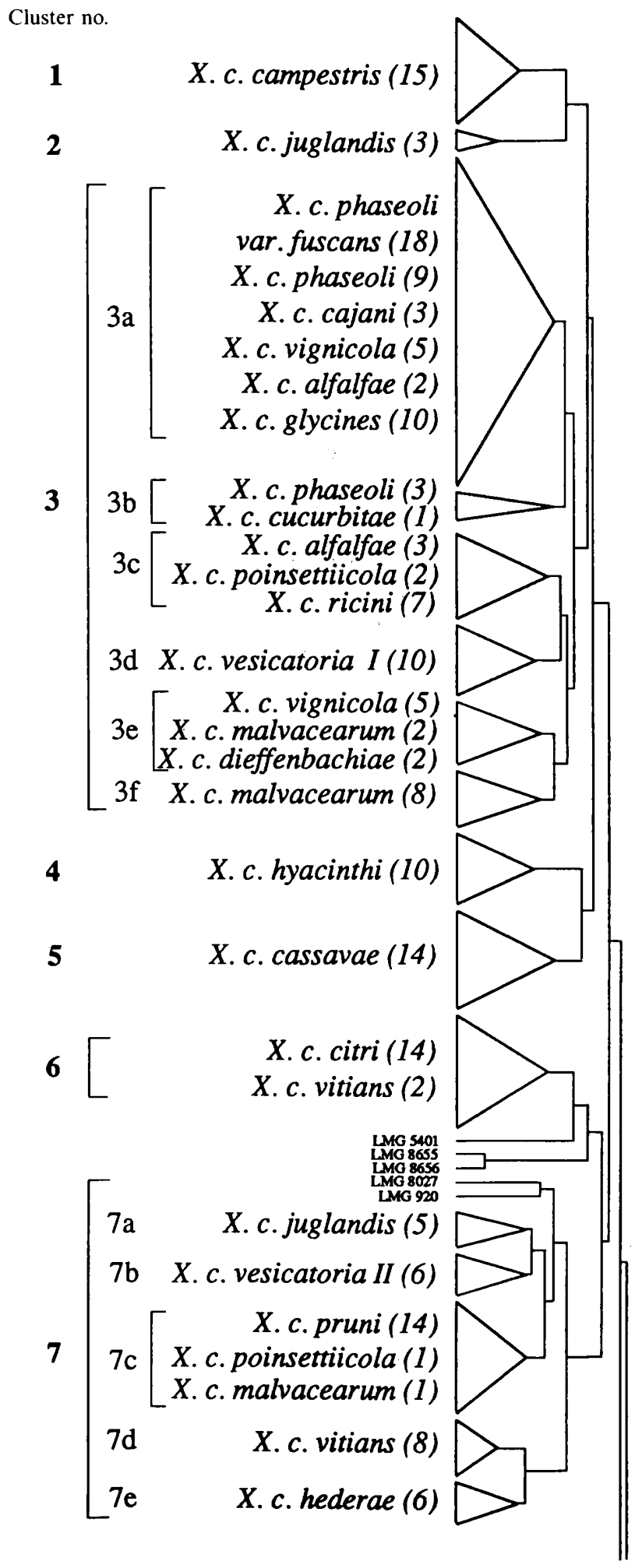




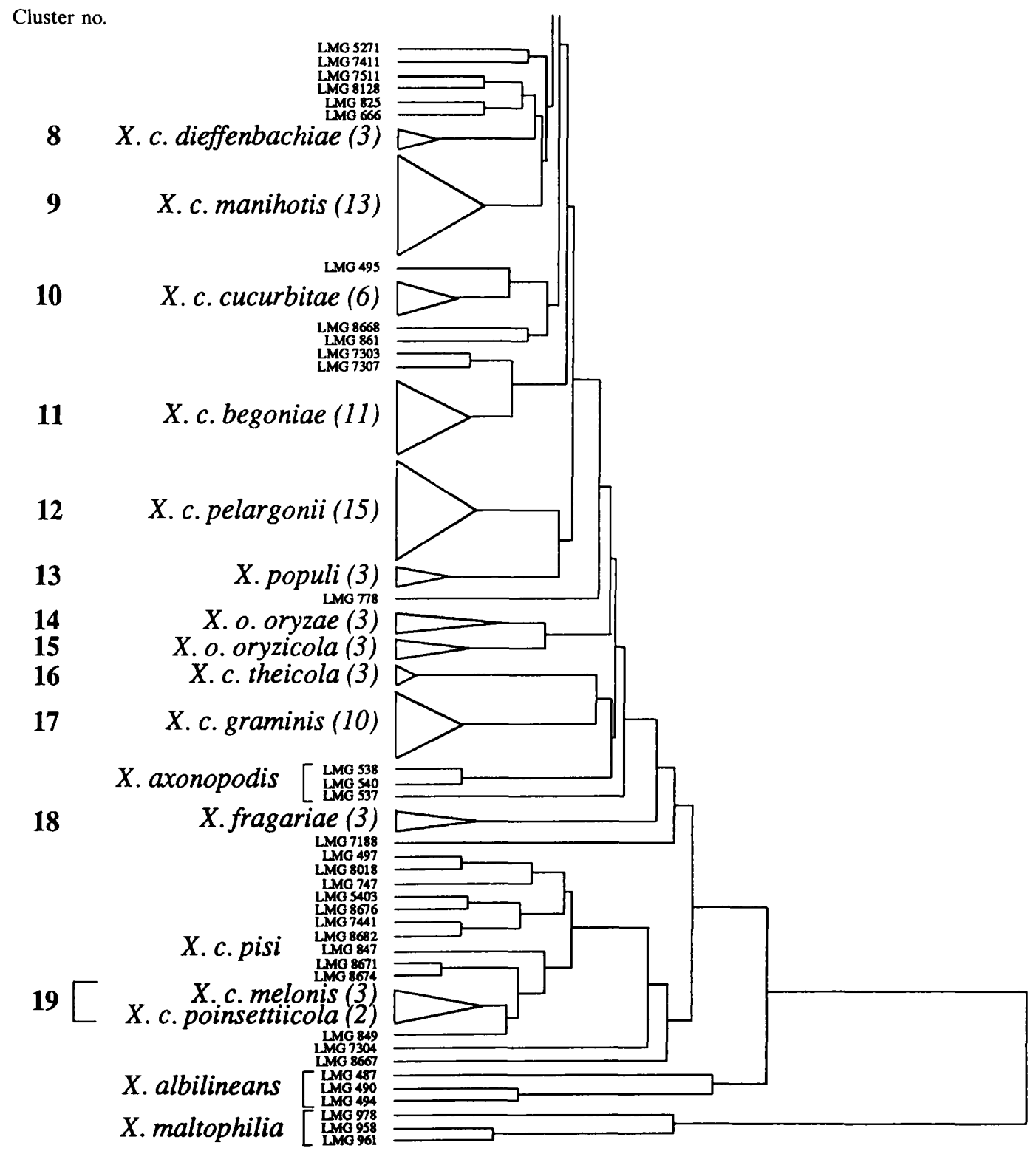

Fig. 1. Simplified dendrogram showing SDS-PAGE clusters of 307 Xanthomonas strains, obtained by average linkage of correlation values. The number of strains per cluster is indicated within parentheses. X., Xanthomonas; X. c., X. campestris;X. o., X. oryzae.

investigated here grouped together at $r=0 \cdot 90$. On the basis of DNA homology, $X$. campestris pv. graminis was found to be related to some other pathovars from grasses and cereals (Kersters et al., 1989).

The three $X$. campestris pv. theicola strains studied have a quite distinct protein profile. These pathogens of tea have only been found in a restricted area in Japan (Uehara \& Arai, 1980), and few strains are available.
$X$. campestris pv. melonis yielded less well resolved protein profiles (Fig. 2), which explains the separation of these strains. Aberrant strains of other pathovars clustered around them, probably due to their diffuse and non-specific profiles.

$X$. campestris pv. poinsettiicola appears to be very heterogeneous in protein profiles; nine strains were spread over three different clusters (3c, 7c and 19), 


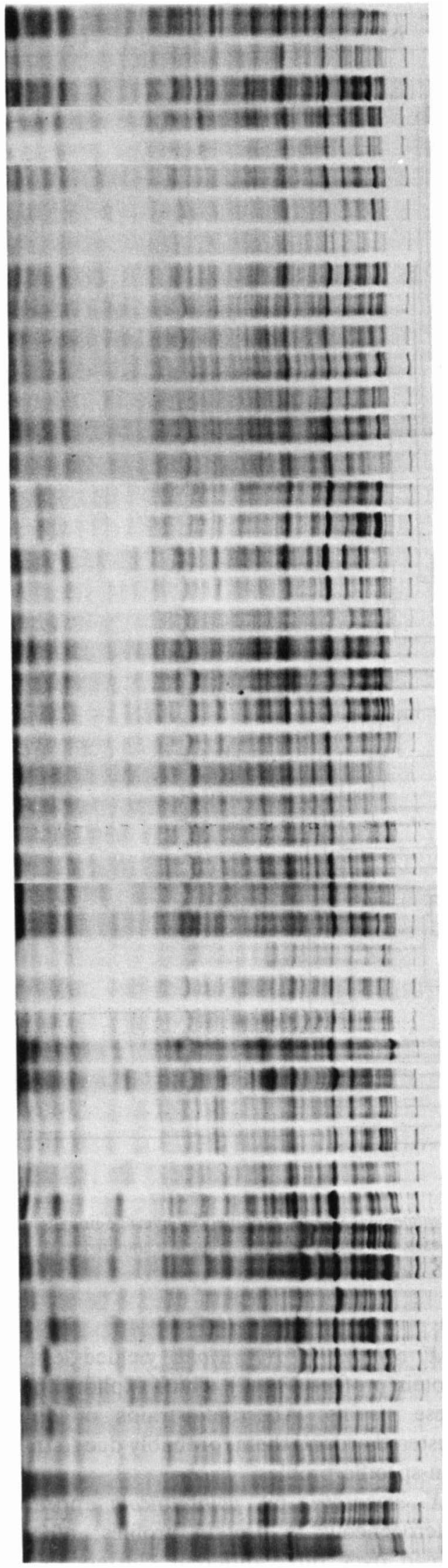

X.c. pv. campestris LMG $568 \mathrm{~T}$

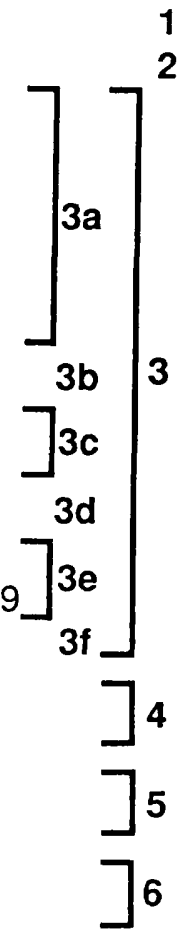

X.c. pv. juglandis LMG 8045

X.c. pv. phaseoli LMG 7455

X.c. pv. cajani LMG 558

X.c. pv. vignicola LMG 839

X.c. pv. alfalfae LMG 8019

X.c. pv. glycines LMG 712

X.c. pv. phaseoli LMG 830

X.c. pv. alfalfae LMG 8080

X.c. pv. ricini LMG 864

X.c. pv. vesicatoria I LMG 910

X.c. pv. vignicola LMG 8752

X.c. pv. dieffenbachiae LMG 7399

X.c. pv. malvacearum LMG 761

X.c. pv. hyacinthi LMG 739

X.c. pv. hyacinthi LMG 8042

X.c. pv. cassavae LMG 673

X.c. pv. cassavae LMG 5246

X.c. pv. citri LMG 682

X.c. pv. vitians LMG 937

X.c. pv. citri LMG 8655

X.c. pv. juglandis LMG 751

X.c. pv. vesicatoria II LMG 911

X.c. pv. pruni LMG 852

X.c. pv. poinsettiicola LMG 5402

X.c. pv. vitians LMG 938

X.c. pv. hederae LMG 733

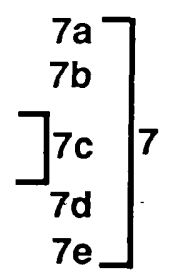

X.c. pv. cassavae LMG 5271

X.c. pv. phaseoli LMG 7511

X.c. pv. dieffenbachiae LMG 695

X.c. pv. manihotis LMG 784

X.c. pv. manihotis LMG 773

X.c. pv. cucurbitae LMG 690

X.c. pv. ricini LMG 861

X.c. pv. begoniae LMG 7303

X.c. pv. begoniae LMG 552

X.c. pv. pelargonii LMG 7314

$X$. populi LMG 5743T

X.o. pv. oryzae LMG 5047T

13

X.o. pv. oryzicola LMG 797

14

X.c. pv. theicola LMG 8684

15

16

$X . c$. pv. graminis LMG 726

17

$X$. axonopodis LMG 538T

$X$. fragariae $L M G$ 708T

18

$X$. albilineans LMG 497

X.c. pv. pisi LMG 847

X.c. pv. melonis LMG 8670

$X$. albilineans LMG 494T

$X$. maltophilia LMG 958T 
whereas four strains were unclustered. This heterogeneity suggests that $X$. campestris pv. poinsettiicola comprises several biological entities. There is no correlation between the types found and the origin of the strains.

Within the main $X$. campestris group, comprising clusters 1 to 12 , a number of homogeneous pathovars were delineated, i.e. $X$. campestris pv. campestris (cluster 1), pv. hyacinthi (cluster 4), pv. cassavae (cluster 5), pv. citri (cluster 6), pv. manihotis (cluster 9), pv. cucurbitae (cluster 10), pv. begoniae (cluster 11) and pv. pelargonii (cluster 12). Among these pathovars, $X$. campestris pv. campestris is very consistent by protein electrophoresis. Fifteen strains of diverse origin grouped at $r=0.90$. It was previously found by Minsavage \& Schaad (1983) that this pathovar is homogeneous on the basis of electrophoresis of membrane proteins. The uniformity of $X$. campestris pv. campestris despite its wide geographical distribution may be explained by the dissemination of this seedborne pathogen (Williams, 1980).

$X$. campestris pv. hyacinthi displayed highly characteristic protein profiles. The pathogen is virulent for Hyacinthus orientalis and has been isolated from nurseries (Kamerman, 1979). Although this pathovar is the oldest known xanthomonad (Wakker, 1883), it has been largely neglected in taxonomic studies, as its occurrence is restricted.

$X$. campestris pv. manihotis and $X$. campestris pv. cassavae are both pathogenic for cassava (Manihot esculentum), but cause different diseases. Previous comparisons between these two pathovars (Maraite \& Weyns, 1979; Dos Santos \& Dianese, 1985; Van den Mooter et al., 1987b) have revealed that they can be differentiated both phenotypically and genotypically. In this study it is shown that both pathovars are distinct from other pathovars as well.

$X$. campestris pv. citri has been the subject of a number of taxonomic and epidemiological studies (Goto et al., 1980; Hartung \& Civerolo, 1987, 1989; Gabriel et al., 1989). This pathovar is a collective designation for xanthomonads causing different diseases of citrus and related hosts (Civerolo, 1984). We have included strains of the most common form of citrus bacterial canker, the A group. The strains constituted a homogeneous cluster, a result consistent with those of RFLP analysis (Hartung \& Civerolo, 1989). Two aberrant strains, LMG 8655 and LMG 8656, isolated from Citrus aurantium in Brazil, probably belong to group $\mathrm{C}$ of citrus bacterial canker disease (Namekata \& Oliveira, 1972).

$X$. campestris pv. cucurbitae strains isolated from pumpkin (Cucurbita maxima) constituted a homogeneous group (cluster 10). The aberrant strain LMG 7479 (subcluster 3b) was isolated from cucumber (Cucumis sativus).

$X$. campestris pv. begoniae and $X$. campestris pv. pelargonii have been studied previously in more detail (Vauterin et al., 1990a) and were characterized as uniform groups. In the present study representative strains of these pathovars were included to locate them in relation to other pathovars. Both pathovars display characteristic protein profiles.

A number of pathovars from leguminous plants (Fabaceae), namely $X$. campestris pv. phaseoli (including var. fuscans), pv. alfalfae, pv. vignicola, pv. cajani and pv. glycines were electrophoretically related to each other. They grouped in cluster 3 , and mostly in subcluster $3 \mathrm{a}$. It is interesting to note that some $X$. campestris pathovars, such as pv. phaseoli, pv. glycines, pv. vignicola and pv. cajani, have overlapping host ranges (Bradbury, 1984). Their phytopathological relatedness and the protein electrophoretic data suggest that the leguminous pathovars in cluster 3 are closely related. This relatedness has been confirmed by DNA-DNA hybridization (Hildebrand et al., 1990; Vauterin et al., 1990b). Strains of $X$. campestris pv. alfalfae and pv. vignicola showed great variability in their protein profiles, which was not correlated with the host or origin of the strains. The pathovar reference strain of $X$. campestris pv. alfalfae, LMG 497, was aberrant. The only leguminous pathovar studied so far which was unrelated to the main cluster 3 was $X$. campestris pv. pisi. However, only one strain of this pathovar is available.

A number of non-leguminous pathovars occurring in subclusters $3 \mathrm{c}$ to $3 \mathrm{f}$ were related to the leguminous group. These included strains of $X$. campestris pv. ricini, pv. vesicatoria, pv. malvacearum, pv. poinsettiicola and $\mathrm{pv}$. dieffenbachiae.

Most of the $X$. campestris pv. ricini strains formed a homogeneous group in subcluster 3c except the nonpathogenic pathovar reference strain LMG 861 and strains LMG 7441 and LMG 8682.

$X$. campestris pv. vesicatoria consists of two substantially different DNA homology groups (subgroup A and subgroup B; see Vauterin et al., 1990b), represented in subcluster $3 \mathrm{~d}$ and subcluster $7 \mathrm{~b}$. The latter group contained the pathovar reference strain LMG 911. No correlation was detected between the electrophoretic groupings and the diverse geographical origin or host plant (pepper or tomato). Two discrete subgroups of $X$. campestris pv. vesicatoria were also found by gas chromatographic profiling of fatty acids (unpublished data). A relationship between $X$. campestris pv. vesicatoria and the pathovars from crucifers (Brassicaceae) $X$.

Fig. 2. SDS-protein electrophoregrams of 50 representative Xanthomonas strains from the clusters obtained by numerical analysis. $X$., Xanthomonas; $X . c ., X$. campestris; X. o., X. oryzae. Note: strain LMG 497 should be named $X$. $c$. alfalfae. 
campestris pv. raphani and $X$. campestris pv. campestris, suggested by Dye et al., 1964, is unlikely on the basis of protein electrophoresis.

$X$. campestris pv. malvacearum constituted a fairly homogeneous electrophoretic group, although this worldwide pathogen of cotton consists of a number of pathological races (Brinkerhoff, 1970). The relatedness of $X$. campestris pv. malvacearum and $X$. campestris pv. vesicatoria subgroup A to xanthomonads from Fabaceae and to each other, as deduced from protein electrophoresis, is consistent with DNA-DNA hybridization data (Vauterin et al., 1990b).

Another cluster of electrophoretically related pathovars was formed by some $X$. campestris pv. juglandis, pv. vesicatoria subgroup $\mathrm{B}$, pv. pruni, $\mathrm{pv}$. vitians and $\mathrm{pv}$. hederae, grouping in cluster 7.

Among these, the protein profiles of $X$. campestris pv. juglandis (subcluster 7a) were very similar to $X$. campestris pv. vesicatoria subgroup B (subcluster $7 \mathrm{~b}$ ). Another type of this pathovar was found in cluster 2, whereas the pathovar reference strain LMG 747 displayed an aberrant protein profile.

SDS-protein profiles of $X$. campestris pv. vitians and $X$. campestris pv. hederae were highly similar (subclusters $7 \mathrm{~d}$ and $7 \mathrm{e}$ ), but pathovar reference strain $X$. campestris pv. vitians LMG 937 and strain LMG 8689 were aberrant. All $X$. campestris pv. hederae strains clustering in group $7 \mathrm{e}$ were isolated from Hedera helix. Two aberrant strains LMG 8667 and LMG 8668 were isolated from Schefflera arboricola and Brassaia actinophylla, respectively, and are clearly misnamed.

Another interesting case is represented by $X$. campestris pv. dieffenbachiae. Although only five strains from this pathovar were tested, the three strains in cluster 8 constitute a well-defined entity, including the pathovar reference strain LMG 695, all isolated from Anthurium in Brazil. The other two strains, isolated from Dieffenbachia in the USA, were closely related to $X$. campestris pv. vignicola and $X$. campestris pv. malvacearum in cluster $3 \mathrm{e}$. It is very likely that the pathogens from Anthurium and Dieffenbachia constitute different biological entities.

In conclusion, it is shown here that a number of distinct protein electrophoretic types exist within $X$. campestris. Some of these groups correspond to described pathovars, others do not. The latter may include strains of different pathovars or may represent only part of a pathovar.

As the knowledge of the homogeneity of a $X$. campestris pathovar is important for both its classification and practical diagnosis, we have drawn particular attention to this aspect of the results. A number of pathovars are obviously homogeneous entities on the basis of protein electrophoresis: $X$. campestris $\mathrm{pv}$. campestris, pv. pelargonii, pv. manihotis, pv. cassavae, $\mathrm{pv}$. hyacinthi, pv. citri (only the Asiatic form), pv. pruni, pv. glycines and pv. graminis Egli \& Schmidt 1982. Others are heterogeneous as they display two or more discrete electrophoretic types. This was the case for $X$. campestris pv. dieffenbachiae, pv. juglandis, pv. alfalfae, pv. poinsettiicola, pv. vesicatoria and $\mathrm{pv}$. vignicola. In most of the latter four cases no correlation was observed between the electrophoretic groupings and the origin of the strains.

It is difficult to evaluate the homogeneity of a pathovar when only a limited number of strains are available, for instance $X$. campestris pv. pisi, pv. cajani, pv. theicola, or when a few strains are more or less aberrant $(X$. campestris pv. begoniae, pv. cucurbitae, pv. malvacearum, pv. hederae and pv. melonis). A problem also arises when the pathovar reference strains is clearly aberrant from the representative group, as was found for $X$. campestris pv. ricini and pv. vitians.

From the results presented here, it is clear that although the taxonomy of Xanthomonas is not settled yet - the SDS-PAGE database of whole-cell protein patterns established in highly standardized conditions can be of great value to identify new and unknown Xanthomonas strains.

L.V. is indebted to the Instituut tot aanmoediging van het Wetenschappelijk Onderzoek in Nijverheid en Landbouw and to the Nationaal Fonds voor Wetenschappelijk Onderzoek (NFWO) for scholarships. The authors acknowledge the NFWO and the Fonds voor Geneeskundig Wetenschappelijk Onderzoek for research grants. Part of the research was carried out in the framework of contract BAP-0138B of the Biotechnology Action Programme of the Commission of the European Communities.

\section{References}

Albritton, W. L., Chen, X. P. \& Khanna, V. (1988). Comparison of whole-cell protein electrophoretic profiles of Haemophilus influenzae: implementation of a microcomputer-mainframe linked system and description of a new similarity coefficient. Canadian Journal of Microbiology 34, 1129-1134.

BRADBURY, J. F. (1984). Genus II. Xanthomonas Dowson 1939, 187. In Bergey's Manual of Systematic Bacteriology, vol. 1, pp. 199-210. Edited by N. R. Krieg \& J. G. Holt. Baltimore: Williams \& Wilkins.

BRINKERHOFF, L. A. (1970). Variation in Xanthomonas malvacearum and its relation to control. Annual Review of Phytopathology 8, 85110.

Civerolo, E. L. (1984). Bacterial cancer disease of citrus. Journal of the Rio Grande Valley Horticultural Society 37, 127-146.

Costas, M., Cookson, B. D., Palsania, H. G. \& Owen, R. J. (1989). Numerical analysis of electrophoretic protein patterns of methicillinresistant strains of Staphylococcus aureus. Journal of Clinical Microbiology 27, 2574-2581.

Dos Santos, R. M. D. B. \& Dianese, J. C. (1985). Comparative membrane characterization of Xanthomonas campestris pv. cassavae and $X$. campestris pv. manihotis. Phytopathology 75, 581-587.

DYE, D. W. (1962). The inadequacy of the usual determinative tests for the identification of Xanthomonas spp. New Zealand Journal of Science 5, 393-416. 
Dye, D. W., Starr, M. P. \& Stolp, H. (1964). Taxonomic classification of Xanthomonas vesicatoria based upon host specificity, bacteriophage sensitivity, and cultural characteristics. Phytopathologische Zeitschrift 51, 394-407.

Dye, D. W., Bradbury, J. F., Goto, M., Hayward, A. C., Lelliott, R. A. \& SCHROTH, M. N. (1980). International standards for naming pathovars of phytopathogenic bacteria and a list of pathovar names and pathotype strains. Review of Plant Pathology 59, 153-168.

EGLI, T. \& SCHMIDT, D. (1982). Pathogenic variation among the causal agent of bacterial wilt of forage grasses. Phytopathologische Zeitschrift 104, 138-150.

El-Sharkawy, T. A. \& Huisingh, D. (1971). Differentiation among Xanthomonas species by polyacrylamide gel electrophoresis of soluble proteins. Journal of General Microbiology 68, 155-165.

ERCOLANI, G. L. (1987). Practical problems with the pathovar scheme in plant quarantine. In Plant Pathogenic Bacteria, pp. 786-793. Edited by E. L. Civerolo, A. Collmer, R. E. Davis \& A. G. Gillaspie. Boston: Martinus Nijhoff Publishers.

Gabriel, D. W., Kingsley, M. T., Hunter, J. E. \& GotTwald, T. (1989). Reinstatement of Xanthomonas citri (ex Hasse) and $X$. phaseoli (ex Smith) to species and reclassification of all $X$. campestris pv. citri strains. International Journal of Systematic Bacteriology 39, 14-22.

Goto, M., TaKahashi, T. \& Messina, M. (1980). A comparative study of the strains of Xanthomonas campestris pv. citri isolated from citrus canker in Japan and cancrosis B in Argentina. Annals of the Phytopathological Society of Japan 46, 329-338.

HARTUNG, J. S. \& Civerolo, E. L. (1987). Genomic fingerprints of Xanthomonas campestris pv. citri strains from Asia, South America, and Florida. Phytopathology 77, 282-285.

HARTUNG, J. S. \& CiVEROLO, E. L. (1989). Restriction fragment length polymorphisms distinguish Xanthomonas campestris strains isolated from Florida citrus nurseries from X.c. pv. citri. Phytopathology 79, 793-799.

Hildebrand, D. C., Palleroni, N. J. \& Schroth, M. N. (1990). Deoxyribonucleic acid relatedness of 24 xanthomonad strains representing 23 Xanthomonas campestris pathovars and Xanthomonas fragariae. Journal of Applied Bacteriology 68, 263-269.

JACKMAN, P. J. H. (1987). Microbial systematics based on electrophoretic whole-cell protein patterns. Methods in Microbiology 19, 209-225.

Jackman, P. J. H., Feltham, R. K. A. \& Sneath, P. H. A. (1983). A program in BASIC for numerical taxonomy of micro-organisms based on electrophoretic protein patterns. Microbios Letters 23, 87-98.

Kamerman, W. (1979). Bacterieziekten in bol- en knolgewassen. Gewasbescherming 10, 103-112.

KENNEDY, B. W. \& ALCORN, S. M. (1980). Estimates of US crop losses to prokaryote plant pathogens. Plant Disease 64, 674-676.

Kersters, K. (1985). Numerical methods in the classification of bacteria by protein electrophoresis. In Computer-assisted Bacterial Systematics, pp. 337-368. Edited by M. Goodfellow, D. Jones \& F. G. Priest. London: Academic Press.

Kersters, K., Pot, B., Hoste, B., Gillis, M. \& De Ley, J. (1989). Protein electrophoresis and DNA:DNA hybridizations of xanthomonads from grasses and cereals. EPPO Bulletin 19, 51-55.

LAEMMLI, U. K. (1970). Cleavage of structural proteins during the assembly of the head of bacteriophage T4. Nature, London 227, 680685.

Leyns, F., De Cleene, M., Swings, J.-G. \& De Ley, J. (1984). The host range of the genus Xanthomonas. Botanical Review 50, 308-356.

MaraITE, H. \& WeYNS, J. (1979). Distinctive physiological biochemical and pathogenic characteristics of Xanthomonas manihotis and $X$. cassavae. In Diseases of Tropical Food Crops, pp. 103-117. Edited by H. Maraite \& J. A. Meyer. Louvain-la-Neuve, Belgium: Université Catholique de Louvain.
Minsavage, G. V. \& SchaAd, N. W. (1983). Characterization of membrane proteins of Xanthomonas campestris pv. campestris. Phytopathology 73, 747-755.

Namekata, T. \& Oliveira, A. R. (1972). Comparative serological studies between Xanthomonas citri and a bacterium causing canker on Mexican lime. In Proceedings of the 3rd International Conference on Plant Pathogenic Bacteria, Wageningen, 14-21, April 1971, pp. 151152.

Pot, B., Gillis, M., Hoste, B., Van de Velde, A., Bekaert, F., KERSTERS, K. \& DE LEY, J. (1989). Intra- and intergeneric relationships of the genus Oceanospirillum. International Journal of Systematic Bacteriology 39, 23-24.

SNeATH, P. H. A. \& SoKal, R. R. (1973). Numerical Taxonomy. The Principles and Practice of Numerical Classification. San Francisco: W. H. Freeman.

STEAD, D. E. (1989). Grouping of Xanthomonas campestris pathovars of cereals and grasses by fatty acid profiling. EPPO Bulletin 19, 57-68.

Uehara, K. \& ARAI, K. (1980). Canker of tea, a new disease and its causal bacterium Xanthomonas campestris pv. theaecola Uehara et Arai pv. nov. Bulletin of the Faculty of Agriculture, Kahoshima University 30, 17-21.

VAN DEN MOOTER, M. \& Swings, J. (1990). Numerical analysis of 295 phenotypic features from 266 Xanthomonas and related strains and an improved taxonomy of the genus Xanthomonas. International Journal of Systematic Bacteriology 40, 348-369.

Van den Mooter, M., Maraite, H., Meiresonne, L., Swings, J., Gillis, M., Kersters, K. \& De LeY, J. (1987a). Comparison between Xanthomonas campestris pv. manihotis (ISPP List 1980) and $X$. campestris pv. cassavae (ISPP List 1980) by means of phenotypic, protein electrophoretic, DNA hybridization and phytopathological techniques. Journal of General Microbiology 133, 57-71.

Van den Mooter, M., Steenackers, M., Maertens, C, Gosselé, F., De Vos, P., Swings, J., Kersters, K. \& De LeY, J. (1987b). Differentiation between Xanthomonas campestris pv. graminis (ISPP List 1980), pv. phleipratensis (ISPP List 1980) emend., pv. poae Egli and Schmidt 1982 and pv. arrhenatheri Egli and Schmidt 1982 by numerical analysis of phenotypic features and protein gel electrophoregrams. Journal of Phytopathology 118, 135-156.

VAN ZYL, E. \& STEYN, P. L. (1990). Differentiation of phytopathogenic Pseudomonas and Xanthomonas species and pathovars by numerical taxonomy and protein gel electrophoregrams. Systematic and Applied Microbiology 13, 60-71.

Vauterin, L., Vantomme, R., Pot, B., Hoste, B., Swings, J. \& KERSTERS, K. (1990a). Taxonomic analysis of Xanthomonas campestris pv. begoniae and $X$. campestris pv. pelargonii by means of phytopathological, phenotypic, protein electrophoretic and DNA hybridization methods. Systematic and Applied Microbiology 13, 166176.

Vauterin, L., Swings, J., Kersters, K., Gillis, M., Mew, T. W., Schroth, M. N., Palleroni, N. J., Hildebrand, D. C., Stead, D. E., Civerolo, E. L., Hayward, A. C., Maraite, H., Stall, R. E., Vidaver, A. K. \& Bradbury, J. F. (1990b). Towards an improved taxonomy of Xanthomonas. International Journal of Systematic Bacteriology 40, 312-316.

Vera Cruz, C. M., Gosselé, F., Kersters, K., Segers, P., Van den MOOTER, M., SWINGS, J. \& DE LEY, J. (1984). Differentiation between Xanthomonas campestris pv. oryzae, $X$. campestris pv. oryzicola and the bacterial 'brown blotch' pathogen on rice by numerical analysis of phenotypic features and protein gel electrophoregrams. Journal of General Microbiology 130, 2983-2999.

WAKKER, J. H. (1883). Vorlaüfige Mitteilungen über Hyacinthenkrankheiten. Botanisches Centralblatt 14, 315-317.

Williams, P. H. (1980). Black rot: a continuing threat to world crucifers. Plant Disease 64, 736-742. 